\title{
SIMBOLISMO TEMPORAL EN GARBA DE JOSÉ MORENO VILLA
}

\author{
ANDRÉS ROMERO JÓDAR
}

Universidad de Zaragoza

\section{RESUMEN}

En 1913, sintiendo el espíritu de incomodidad europea por la cercanía de la explosión de la Gran Guerra, aparece publicada bajo el título de Garba, la primera colección/libro de poemas de José Moreno Villa, poeta y pintor que se mueve entre Modernismo y vanguardias de comienzos del siglo XX en las letras españolas. Garba ha sido considerada como una obra modernista de juventud, dentro de un propio «Modernismo a la andaluza», entendiendo modernismo como un «movimiento» sincrético, híbrido de diferentes tendencias, desde el parnasianismo al simbolismo, pasando por el decadentismo del mal de fin de siglo. De una forma general, sin embargo, puede verse esta creación como una obra de su tiempo en su contexto europeo, obra propia de un momento histórico y social de crisis en los valores tradicionales y cambios en las concepciones temporales.

Sin olvidar su contexto español, este breve análisis tiene como objetivo destacar la unidad de la obra como conjunto, basada en una estructura cíclica cercana a la percepción del tiempo de la naturaleza - frente al tiempo mecanizado-, intentando escapar de ese espíritu alienado que conlleva la industrialización del comienzo de siglo. Es decir, Garba puede verse como una obra reflejo de las inquietudes en la percepción temporal que sufrían otros autores coetáneos más allá de las fronteras españolas.

Palabras clave: José Moreno Villa, Garba, Modernismo español, Modernismo europeo, poesía española del siglo xx, estructura cíclica.

\section{TIME SYMBOLISM IN GARBA, BY JOSÉ MORENO VILLA}

\section{ABSTRACT}

In 1913, feeling the European uneasy spirits due to the threatening closeness of the Great War's outbreak, the first collection/poem book of José Moreno Villa (poet and painter who stands in between Modernism and Avant-gardes at the beginning of the Xx century of the Spanish literature) appears published with the title of Garba. This work has been considered to be a modernist book of youth, inside a Modernism «a la andaluza,» conceiving Modernism as a syncretic «movement», hybrid from different trends, from Parnasianism to Symbolism, suffering from the decadentism of the fin de siècle's fits. In a general sense, however, Garba can be seen as a work of its times in its European context, a work proper to a historical and social moment of crisis in the traditional values and changes in time perceptions.

Without forgetting about its Spanish context, this short analysis has the aim of highlighting the unity of the work as a whole, a unity which is based on a cyclical structure close to the 
time perception of Nature — against mechanized time_- trying to break free from that alienated feeling which the industrialization of the beginning of the century conveys. Therefore, Gar$b a$ may be seen as a work which reflects all the concerns about time perception which other contemporary authors were suffering far beyond the Spanish frontiers.

Key words: José Moreno Villa, Garba, Spanish Modernism, European Modernism, Spanish poetry of the $\mathrm{xx}$ century, Cyclical structure.

En 1913, sintiendo el espíritu de incomodidad europea por la cercanía de la explosión de la Gran Guerra, aparece publicada bajo el título de Garba, la primera colección/libro de poemas de José Moreno Villa (18871955), poeta y pintor cuyo contexto literario es difícil de clarificar, al moverse entre Modernismo y vanguardias de comienzos del siglo XX en las letras españolas. Garba ha sido considerada como una obra modernista de juventud, dentro de un propio «Modernismo a la andaluza» (Carnero, 1989: 182), entendiendo modernismo como un «movimiento» sincrético, híbrido de diferentes tendencias, desde el parnasianismo al simbolismo, pasando por el decadentismo del mal de fin de siglo. De una forma general, sin embargo, puede verse esta creación como una obra de su tiempo en su contexto europeo, obra propia de un momento histórico y social de crisis en los valores tradicionales y cambios en las concepciones temporales.

Sin olvidar su contexto español, este breve análisis tiene como objetivo destacar la unidad de la obra como conjunto, basada en una estructura cíclica cercana a la percepción del tiempo de la naturaleza - frente al tiempo mecanizado-, intentando escapar de ese espíritu alienado que conlleva la industrialización del comienzo de siglo. Es decir, Garba puede verse como una obra reflejo de las inquietudes en la percepción temporal que sufrían otros autores coetáneos más allá de las fronteras españolas.

A nivel europeo, el final del siglo XIX y comienzos del XX es un periodo de cambios sociales bruscos, promovidos por el movimiento de industrialización y centralización en las ciudades. Se trata de un momento de crisis de conciencia personal y filosófica, debida a diferentes e importantes acontecimientos. Por un lado, se produce la asimilación de la degeneración que suponía la aceptación de los postulados darwinistas ya planteados a mitad del siglo XIX. En 1859, Charles Robert Darwin publica El origen de las especies, refutando las teorías creacionistas, basadas en el relato bíblico de la creación de la existencia, e influenciando a toda Europa con las ideas acerca de la degeneración. Aparecen teóricos apoyando esta concepción degenerativa, como Max Nordau en Alemania; Benedict Morel, quien acuñó el término dégénérescence en 1857, en Francia; o Cesare Lombroso en Italia (Ledger, 2000). Estos postulados llevarían a la aparición de las ideas del llamado darwinismo social, encabezadas por el filósofo Herbert Spencer, quien enarbolaría el lema de la «supervivencia de 
los más aptos» ${ }^{1}$ (1898: 58). A su vez estos principios sirven de unión con la figura emblemática de la degeneración a final del XIX, Friedrich Nietzsche (1844-1900), y su exposición del superhombre que representará los instintos de competitividad y supervivencia.

Por otro lado, también a finales de siglo y, sobre todo, al comienzo del nuevo, podemos encontrar otra corriente esencial en el pensamiento europeo. Continuando y personalizando las enseñanzas de Jean-Martin Charcot en La Salpêtrière ${ }^{2}$ de París, Sigmund Freud y sus seguidores proponen las bases del Psicoanálisis, donde lo reprimido, el $i d$, lo oculto de nuestra mente, se impone sobre nuestros actos más básicos. De este modo, la mente se convierte en algo incontrolable, fuente de misterio e intranquilidad, ya que todos poseemos un lado irracional que nos impulsa a comportarnos de una manera determinada (Tallaferro, 1995).

A todo esto podríamos añadir la nueva concepción del tiempo para las ciencias en general y la Física en particular. Se ofrece una relativización del tiempo y espacio al compararse la energía con la materia en la celebérrima ecuación $e=m c^{2}$ que Albert Einstein publicara en 1905 (Einstein, 2004).

De este modo, podemos apreciar cambios importantes en la concepción del mundo y de la realidad en todo el pensamiento europeo. Como bien señala Lily Litvak, se puede decir que en esos años del cambio de siglo hay una clara expresión de incomodidad e insatisfacción tanto con el materialismo que trae la industrialización y la sociedad consumista de cultura de masas, como con el racionalismo exacerbado y la impersonalidad de la sociedad burguesa, «a la vez que se manifiestan ciertas inquietudes metafísicas, espirituales y sociales» (1990, pág. 16). Se puede afirmar que el final del siglo XIX supone un periodo de incomodidad para la cultura y el pensamiento occidentales.

Evans Lansing Smith, en su libro Rape and Revelation, the Descent to the Underworld in Modernism, se basa en esta situación de crisis ideológica para formular su propuesta acerca de la «nueva» estructura que van a tomar las obras literarias en este periodo. Se va a buscar una estructura cíclica, cercana a la mítica, para recuperar ese sentido de la vida perdido por los recientes cambios y catástrofes mundiales. Smith afirma que:

The composition of [Modernists] Works [based on the «mythic method»] tmends to coincide with a crisis in the writers' lives, analogous to the descent to the underworld. This breakdown in the inner sphere is reflected outwardly by the

${ }^{1}$ «Survival of the fittest» (Spencer, 1898: 58).

${ }^{2}$ El hospital parisino de La Salpêtrière se convirtió en centro de peregrinaje para los científicos de las emergentes disciplinas de la Psicología, la Psiquiatría y la Neurología. A finales del siglo XIX, bajo la tutela de Jean-Martin Charcot, estudiaron y desarrollaron sus ideas nombres ilustres de las neurociencias tales como Pierre Janet, William James o Sigmund Freud. 
cultural catastrophe of World War I, and by certain other developments in the arts and sciences of the times. Hence the myth gives that shape and significance to the works and lives of the Modernists (1990: 1) $)^{3}$.

Smith vincula la crisis interna del individuo con la catástrofe cultural externa de la Primera Guerra Mundial para justificar la necesidad de imponer el patrón unitario del mito en las obras modernistas. De este modo, en el interior de la mente alienada modernista, aterrada por la degeneración cultural y filosófica, enterrada bajo esas catástrofes culturales, parece reposar la creencia en la posibilidad de regresar a la situación inicial de armonía unitaria — sugerida por la circularidad de la estructura mítica一. El retorno al mito, a la estructura circular natural, sugiere la posibilidad de encontrar un escape optimista frente a la decadencia general de comienzos del siglo Xx y que culminará con el comienzo de la Gran Guerra en 1914.

Es dentro de esta tendencia europea donde podemos analizar la primera obra de José Moreno Villa, Garba (1913). De forma general, Rosa Romojaro apunta que este libro aborda temas «psicológicos, moralizadores, líricos, históricos, descriptivos, filosóficos, irónicos y españoles [,] como reflejo de sus propias circunstancias o inquietudes vitales [de Moreno Vi1la]» (2004: 21). A estos temas, podríamos añadir la concepción del tiempo como un elemento importante en la lectura de Garba. Como se ha dicho antes, el objetivo de este breve ensayo es resaltar la unidad de la obra como conjunto dentro de estos postulados modernistas de búsqueda de unidad circular. Esta concepción temporal se acerca a la percepción del tiempo de la naturaleza - frente al tiempo mecanizado de las crecientes urbes industrializadas-, intentando huir de esa sensación de alienación que conlleva la industrialización. Por medio de un símbolo de esperanza en un proceso infinito - la espiga-, Garba $a^{4}$ parece estar concebido de manera que represente el ciclo de la naturaleza, el movimiento del sol desde el amanecer hasta el anochecer, buscando «algo eterno, permanente» (En Córdoba, Moreno Villa, 1998: 123).

Garba se divide en cinco partes: «Espigas», «Tres momentos del parque de Málaga», «Las sugestiones del mar», «Recreos»y «Espigas negras». Lo interesante de esta división es el esquema temporal que parece subya-

\footnotetext{
3 «La composición de obras [modernistas basadas en el método mítico] tiende a coincidir con una crisis en las vidas de los escritores, análoga al descenso al inframundo. Esta crisis en la esfera interna se refleja en el exterior por la catástrofe cultural de la Primera Guerra Mundial, y por otros ciertos desarrollos en las artes y ciencias del momento. Así, el mito da esa forma y significación a las obras y vidas de los modernistas» (1990: 1, mi traducción).

${ }^{4}$ El título, Garba, se lo propuso el poeta Enrique de Mesa, entonces secretario del Ateneo de Madrid, muy frecuentado por Moreno Villa, y donde entra en contacto con otros poetas como Ramón Pérez de Ayala o Enrique Díaz-Canedo.
} 
cer al considerar la obra en conjunto. Si partimos viendo un simbolismo en el título, Garba, que literalmente es un conjunto de sarmientos, cañas, mieses, etc., mayor que el manojo y menor que el haz, podemos apreciar un nuevo sentido en el libro entero. La obra es garba, conjunto de espigas, y por lo tanto, los poemas que forman el libro serán esas espigas.

De este modo, a lo largo de toda la obra está presente la imagen del poema como espiga, con la doble significación temporal que semejante símbolo contiene. Es decir, el poema-espiga es a la vez comienzo y final de un ciclo, de un periodo marcado por el tiempo de la naturaleza. Por un lado, la espiga es el final en el crecimiento de la semilla, e implica una inminente separación de la tierra al ser cosechada. Por otro lado, la espiga es también imagen de futuro al ver su próxima transformación en un producto más elaborado. Como señala Ciplijauskaité, hay un «énfasis en las posibilidades latentes, es decir, el futuro» (1999: 37), aunque no con un movimiento de ascensión, como esta autora afirma, sino con un movimiento cíclico que nunca termina. Con este doble sentido de final y comienzo, es importante el poema que abre el texto:

\section{ESPIGAS}

Sobre dorados juncos, el pan en embrión se balancea al ritmo seráfico del viento... Cada grano de trigo lleva en el corazón una cerda emblemática que mira al firmamento (1998: 79).

La espiga es «pan en embrión» (v. 1), es su propia potencialidad de futuro, llevada por el «ritmo» (v. 2) que marca la naturaleza, fuera del control temporal humano, el tiempo de la máquina. Además, dentro de cada espiga - que se puede ver como imagen del poema- hay un grano de trigo - su «alma»- que escruta el infinito en busca de significado.

Se establece así una dicotomía entre el título de la obra y el primer poema. Garba implica final, cosecha, recogida de las mieses - aunque también principio de la segunda fase del maíz-, mientras que espigas remonta a ese momento de unión con la tierra, el crecimiento de semilla a espiga. De este modo, ya en el principio de su obra, Moreno Villa intenta conciliar esos opuestos y establece las bases para entender el tiempo de su obra, dentro de una concepción cíclica de la existencia.

Por otro lado, dándole unidad global a la obra, hay que considerar la última parte, «Espigas Negras», con ese mismo sentido de oposición frente a la primera parte, «Espigas». En «Espigas» tenemos la luz del sol amaneciendo «sobre dorados juncos» (ESPIGAS, v. 1, pág. 79), buscando el alma, la esencia de las cosas — como en ANTE LA CATEDRAL DE LEÓN, pág. 79, donde se hace una interesante asociación entre alma y verso, ya que son versos de nueve sílabas y nueve veces se repite la palabra «alma»-, acabando con una SENSACIÓN DE OCASO (pág. 91) que nos une al impre- 
sionismo, para entrar en una noche de estío de la mano de EL NIETO DE DON QUIJOTE (pág. 94). Se completa así un ciclo de amanecer-anochecer, que se queda abierto por medio de una breve COPLILla (pág. 96).

Sin embargo, en «Espigas Negras», última parte del libro, encontramos el anochecer de Garba. Todo aparece a «contra sol» (LA HOMBRADA, v. 2, pág. 112); los viejos trabajadores duermen y sueñan, siendo «eco de otros siglos» (LO FATAL, v. 23-24, pág. 112); simbolizados en el color azabache de EL TORO DE LIDIA (pág. 115), durante una «noche cálida» (LO DE LA SERRANíA, v. 1, pág. 115).

Es decir, podemos observar que la obra está pensada como un todo, como un ejemplo de ciclo natural de días y noches englobados entre un amanecer — «Espigas»— y un anochecer — «Espigas Negras»-, dando así una sensación de unidad y de estructura natural. Por supuesto, para que dicho simbolismo funcione y cobre significado, se tiene que contraponer esta concepción temporal a la percepción mecanizada del tiempo que ha venido con la revolución industrial y la deshumanización del trabajador en favor de la máquina.

Se podría decir que la revolución industrial y la mecanización del trabajo en la sociedad de fin de siglo tienen dos consecuencias claves en la concepción del tiempo y del trabajador. Por un lado, está el tiempo del trabajo, el de la máquina, regido por las horas del reloj —convirtiéndose éste en uno de los grandes símbolos de angustia existencial y de elemento contra el que luchar, como podemos ver en los caligramas de Guillaume Apollinaire, como «La cravate et la montre» (Apollinaire, 1971)—, siendo así el tiempo de la burguesía y del dinero. Frente a éste, encontramos el tiempo natural, de la naturaleza, con sus ciclos temporales y se percepciones de luz y color, el tiempo mítico, el de nacimiento-muerte-regeneración/resurrección.

Por otro lado, el control que ejerce el tiempo de la máquina sobre el ser humano hace que éste se convierta en una pieza más de la maquinaria industrial — como se puede ver en la maravillosa visión de Charles Chaplin en Tiempos modernos (1935)—, o en un autómata decadente, mitad humano, mitad máquina — como en la futurista Metrópolis (1927) de Fritz Lang, exponente del expresionismo alemán, donde se crea una cierta actualización del mito de Frankenstein que llegará hasta nuestros días en la concepción del mundo ciberpunk de William Gibson o Shirow Masamune-.

Ese sentimiento de ser mecanizado provoca una sensación de alienación en el individuo, un sentimiento de extrañeza y decadencia. Ese tiempo de la máquina elimina el sentido metafísico de la vida, lo que Moreno Villa expresa en EL TAPIZ PERSA (1998: 84):

$$
\begin{gathered}
11[\ldots] \text { Tu pie, pobre pie de ciego } \\
\text { que no sabe a dónde va, } \\
\text { ni por qué es dura la tierra, }
\end{gathered}
$$




\section{ni por qué tiene que andar... \\ 15 Pie de ciego, que ha pintado de carmín la blanca paz del sendero $[\ldots]$.}

Aquí se expresa la falta de sentido y la incapacidad de encontrar significado a la vida. El caminante, «ciego» (v. 11), ignora tanto su destino como el motivo de su existencia. Sólo sabe que tiene que seguir trabajando/andando como un autómata, hasta cubrir con sangre el camino (vv. 16-17). Este poema es ejemplo de la visión decadente de la vida y del tiempo moderno, donde el contacto con los ciclos de la naturaleza se ha perdido, siendo sustituido por el férreo control del reloj.

Contra este tiempo mecanizado Moreno Villa grita y explora ${ }^{5}$, entendiendo, en sus propias palabras, la poesía como confesión a gritos. Como él mismo señala, en el momento de composición de Garba, «[su] principal y casi única creencia afirmaba que la poesía era sobre todo confesión. Abrir el pecho ante los otros, confesarse a gritos como los antiguos cristianos, [le] parecía misión de poeta, noble y bella de por sí» (1998: 5). Como un don Quijote desquiciado, se lanza a la lucha contra esos molinos monótonos que destruyen la individualidad del ser humano. PIRUETA (pág. $85)$ es un claro ejemplo de la declaración de principios del poeta:

$1 \quad ¡$ Las alas de aquel molino

te están diciendo constancia

y tú no quieres saber

más que de locuras, alma!

5 -No sé si quiero locuras,

pero no quiero ser aspa

mecánica de un molino

que sin voluntad trabaja.

Quiero ser como ese mirlo

10 que se chapuza en el agua, que pía cuando es su gusto, que a placer abre sus alas, que se sublima en los cielos, y que, cuando quiere, baja...

15 iY siento un hondo desprecio por el chisme de las aspas, que a su tic-tac condenado se reduce y amilana!

La segunda estrofa (vv. 5-8) es claramente significativa. El alma joven del poeta aboga por la libertad natural del «mirlo», asociando las aves

${ }^{5}$ La exploración, según Ana Rodríguez-Fischer, es la característica primordial que situaría a José Moreno Villa dentro de las corrientes vanguardistas. Según esta autora, «para José Moreno Villa, en esa condición de exploración y búsqueda reside el genuino espíritu vanguardista» (1999: 20). 
con la esfera espiritual, frente al monótono devenir temporal de las aspas del molino, carente de voluntad o individualidad. Se lucha contra ese autómata que pretende imponer el tiempo mecanizado, haciendo así una apología de la vida natural, de la concepción mítica, cíclica de la vida en comunidad con la naturaleza.

Otro ejemplo de ese espíritu alienado modernista, o al menos producido por la modernidad, es el poema SONSONETE (pág. 88), destacando la estrofa tercera:

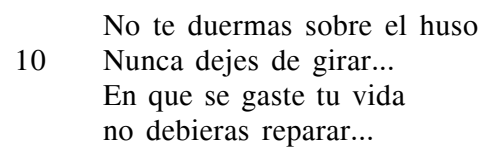

Este poema parece plantearse como los consejos que da un trabajador a otro, ambos englobados dentro de ese tiempo del trabajo al que se ha hecho mención previamente. Ya el título es significativo: Sonsonete es un sonido rítmico, monótono y generalmente molesto, producido por cualquier clase de golpes. Es así la oposición a la poesía y al espíritu libre del mirlo del poema anterior. Es una pieza más de ese mundo industrializado. Los versos destacados aquí reflejan la resignación frente a ese control de la máquina: el hombre-autómata no debe preocuparse por el pasar de los años, sólo le debe importar que la máquina nunca deje de girar, como una ominosa rueda de la fortuna que lleva directamente hacia la muerte y el olvido.

Los viejos oficios, como el labrador, o la vieja con su rueca, se ven así como parte de la tradición que ha muerto con la entrada de la maquinalización de las industrias. Ahora son sueños de otras épocas pasadas, «ecos de otros siglos» como señala en LO FATAL (pág. 113).

Por último, la modernidad trae también consigo una división entre la percepción del tiempo «real», mecanizado por medio del reloj, y el tiempo subjetivo del individuo - lo que Bergson llamará la durée réelle, o tiempo medible, en oposición a la durée interne, o tiempo interior (1968)—. Esta percepción del tiempo individual se nutre de recuerdos y memorias del pasado - como ejemplifica el excelente caso de Marcel Proust en el primer volumen de A la recherche du temps perdu (1919-1927), evocando la infancia del protagonista por medio de una magdalena-, pero siempre sin conexiones racionalizables, sino por medio de impresiones externas - como el extremadamente complejo ejemplo de Benji en The Sound and the Fury (1929) de William Faulkner- . De este modo expresa Moreno Villa su capacidad poética, en su poética de 1932:

El fenómeno poético es un estado de gracia. No sé cómo poder dibujarlo con la pluma. Yo sí que me desligo totalmente de lo circundante y que penetro en un zona luminosa y sorda, donde la situación de mi ánimo o la intención inicial que 
traía al penetrar en ella va cuajando o expresándose gracias a la baraja de posibilidades, recuerdos, asociaciones de la fantasía (1998: 9).

Por medio de impresiones en los sentidos, se llega a esa abstracción que lleva a los recuerdos y las asociaciones. El tiempo de percepción unitaria de la realidad pertenece al mundo/ritmo de la máquina, de la sociedad industrial. Por eso, el tiempo verdadero, el que le interesa al poeta, a la poesía, y por tanto, al alma, sólo se puede percibir a través de impresiones sensoriales que evocan situaciones y recuerdos. Se rompe así con el tiempo lineal favoreciendo la estructura mítica/cíclica de la obra. Además, esas impresiones sensoriales apuntan a la faceta de pintor de Moreno Villa, y como él mismo dice, «Garba resultó como un muestrario de reacciones varias ante las más variadas situaciones» (1998: 38).

El mejor ejemplo de cómo afectan las impresiones sensoriales al poeta lo encontramos en la segunda parte del libro, titulada «Tres momentos del parque de Málaga». Una vez más se percibe el ciclo de la naturaleza representado en la poesía, pasando del mediodía en «el muelle, calcinado de sol» (LOS VAGOS DUERMEN, v.1, pág. 97), al atardecer, reverdeciendo el jardín (LOS NIÑOS TRIUNFAN, v. 2, pág. 97), hasta la noche que cierra el ciclo natural (CUPIDO VENCE y NOCTURNO, pág. 98). Empleando una técnica impresionista, se dan pinceladas sobre las diferentes sensaciones que causa el paso del tiempo en el mismo lugar sobre la percepción del poeta.

Para concluir, José Moreno Villa emplea la poesía en esta obra como mecanismo de escape de una sociedad alienada. Como él mismo señala, «la psique, lo mismo que el cuerpo humano y la máquina, necesita un escape cuando la presión interior es demasiado alta [...]. Sin el escape de la poesía, hubiera tenido que ingresar en una orden monástica siendo joven (1998: 18). En esta primera obra, Garba, de 1913, el joven poeta parece intentar escapar de esos cambios en la percepción de la realidad que ha traído la modernidad. Siguiendo una estela europea que llevará a T. S. Eliot y su The Waste Land (1922) o a James Joyce y su Ulysses (1922), Moreno Villa recurre al esquema y concepción cíclica de orden mítico para tratar de infundir significado a su obra y a su vida, en una época en la que el ser humano se ha convertido en un autómata al servicio de la máquina.

\section{REFERENCIAS BIBLIOGRÁFICAS}

APOLLINAIRE, Guillaume. Calligrammes: Poèmes de la paix et de la guerre (1913-1916). Paris: Gallimard, 1971.

BERGSON, Henri. Durée et simultanéité: à propos de la théorie d'Einstein. Paris: Presses Universitaires de France, 1968 (1922).

CARNERO, Guillermo. Las armas abisinias: Ensayos sobre literatura y arte del siglo XX. Barcelona: Anthropos, 1989.

CIPLIJAUSKAITÉ, Biruté. De signos y significaciones. I. Juegos con la Vanguardia: poetas del 27. Barcelona: Anthropos, 1999. 
CHAPLIN, Charles. Tiempos modernos. Estados Unidos : United Artists, 1935.

DARWIN, Charles. The Origin of Species by Means of Natural Selection, or, The Preservation of Favoured Races in the Struggle for Life. Harmondsworth: Penguin, 1985 (1859).

EINSTEIN, Albert. Cien años de relatividad: los artículos clave de Albert Einstein de 1905 a 1906. RUIZ DE ELVIRA, Antonio (trad.). Tres Cantos: Nivola Libros y Ediciones, 2004.

ELIOT, Thomas Stearns. The Waste Land. Londres: Faber and Faber, 1980 (1922).

FAULKNER, William. The Sound and the Fury,.Londres : Vintage, 1995 (1929).

GIBSON, William. Neuromancer. Londres: HarperCollins, 1995 (1989).

JOYCE, James. Ulysses. Harmondsworth: Penguin, 1978 (1922).

LANG, Fritz. Metrópolis. Alemania: UFA, 1927.

LEDGER, Sally y LUCKHURST, Roger. The Fin de Siècle. A Reader in Cultural History c.1880 - 1900. Oxford y New York: Oxford University Press, 2000.

LITVAK, Lily. España 1900. Modernismo, anarquismo y fin de siglo. Barcelona: Anthropos, 1990.

MASAMUNE, Shirow. Ghost in the Shell SIMÓ, Victoria (trad.). Barcelona: Planeta de Agostini, 1991.

MORENO VILLA, José. Poesías completas. PÉREZ DE AYALA, Juan (ed.). México y Madrid: Centro de Estudios Lingüísticos y Literarios y Publicaciones de la Residencia de Estudiantes, 1998.

NIETZSCHE, Friedrich. Obras completas. Vol. 4, La voluntad de dominio; El ocaso de los ídolos; Ecce Homo. OVEJERO Y MAURY, Eduardo (ed.). Madrid: Aguilar, 1967.

PROUST, Marcel. Por el camino de Swann. SALINAS, Pedro (trad.). Barcelona: Orbis, 1982 (1919-1927).

RODRÍGUEZ-FISCHER, Ana. Prosa española de vanguardia. Madrid: Castalia, 1999.

ROMOJARO, Rosa. Lo escrito y lo leído: ensayos sobre literatura y crítica literaria. Barcelona: Anthropos, 2004.

SMITH, Evans Lansing. Rape and Revelation. The Descent to the Underworld in Modernism, Lanham, New York \& Londres: University Press of America, 1990.

SPENCER, Herbert. The Principles of Biology. Vol. II. New York: D. Appleton and Company, 1898.

TALlAFERRO, Alberto. Curso básico de Psicoanálisis. México: Paidós, 1995.

Fecha de recepción: 22 de mayo de 2008

Fecha de aceptación: 2 de enero de 2009 